10

\title{
An agent based control system for a model factory
}

\author{
A.J.R. Zwegers, H.J. Pels, R.L.J. Schrijver, R.J. van den Berg \\ Department of Technology Management \\ Eindhoven University of Technology, Pav. C10 \\ P.O. Box 513, 5600 MB Eindhoven, the Netherlands \\ Tel: +31-40-2472671 Fax: +31-40-2436492 \\ Email: A.J.R.Zwegers@tm.tue.nl
}

\begin{abstract}
The objective of this paper is to present the design of an agent based system for a specific model factory. A manufacturing system can be seen as a collection of autonomous, problem solving agents which interact when they have interdependencies. A concurrent programming formalism is used for the specification of the agent based system. Agents have the possibility to subcontract jobs. Compared to a previously specified heterarchical control architecture, the agent based system is more robust and flexible. The suitability of an agent based approach is dependent on characteristics of the production system, such as uncertainty in product specifications.
\end{abstract}

\section{Keywords}

Agent Based Systems, Control Architecture, Heterarchical Control, Shop Floor Control.

\section{INTRODUCTION}

Current production management architectures show significant deficiencies in controlling the complexity and the uncertainty that is typical of manufacturing systems. In manufacturing systems, the predominant architectural paradigm has up to now been hierarchical. Because of its mechanistic and deterministic approach, the hierarchical paradigm has numerous defects in coping with uncertainty and 
with the rapidly evolving scenario that characterises today's manufacturing environments. In this paper, we concentrate on another approach that is derived from Distributed Artificial Intelligence, and that is based on the concept of distributed, autonomous agents.

The objective of this paper is to present the design of an agent based system, and to compare the characteristics of the agent based architecture with those of a previously specified control architecture. In addition, we mention some considerations in designing an agent based system. We apply the concepts of the agent based approach in a model factory of a printed circuit board assembly and test line. The architecture is specified by means of a concurrent programming technique, which is suitable for the specification of distributed control architectures. The agent based design is subsequently compared to another heterarchical control system, which has previously been designed and implemented in the model factory.

The paper is organised as follows. In the next section, the agent based concept is explained in more detail. After this, we shortly describe the specification language we have applied. Then, the actual design of an agent based control system is given, together with some considerations that have to be treated during the design process. Finally, we discuss the agent based control system compared to the heterarchical system, and we touch upon the situations in which an agent based control system proves most suitable.

\section{AGENT BASED SYSTEMS}

Strong similarities can be found between the characteristics of agents and those of current manufacturing systems. Manufacturing processes are highly dynamic and unpredictable; it is difficult to completely separate the planning and sequencing of required activities from their execution. Any detailed time plans are often disrupted by unpredictable delays and other unanticipated events. As a result, a tendency exists within manufacturing systems to decentralise the ownership of the tasks, information, and resources involved in the various processes. Different groups within manufacturing systems become relatively autonomous: how their resources are consumed, by whom, at what cost, and in which time frame lies within their own prerogative.

Given these characteristics, it is quite natural to model the processes in a manufacturing system as a set of autonomous, problem solving agents which interact when they have interdependencies. In such a context, an agent can be seen as an encapsulated problem solving entity that exhibits the following properties:

- Autonomy: agents perform the majority of their problem solving tasks without the direct intervention of other agents; they control their own actions and their own internal state.

- Social ability: agents interact, when they deem appropriate, with other agents in order to complete their problem solving and to help others with their tasks. 
This implies that agents have, as a minimum, a means by which they can communicate their requirements to others and an internal mechanism to decide what and when social interactions are appropriate (both in terms of generating requests and judging incoming requests).

- Proactiveness: agents take the initiative where appropriate.

- Responsiveness: agents perceive their environment and respond in a timely fashion to changes that occur in it (Jennings, et al., 1996).

Each agent is able to perform one or more services or tasks. If an agent requires a service that is managed by another agent, it cannot simply instruct the other agent to start the service; agents are autonomous, and control dependencies between them do not exist. Instead, the agents must come to a mutually acceptable agreement about the terms and conditions under which the desired service will be performed. The mechanism for making these agreements is negotiation, a joint decision making process in which the parties verbalise their demands and then move towards agreement by a process of concession.

To negotiate with one another, agents need a protocol that specifies the role of the current message interchange, e.g. whether the agent is making a proposal or responding with a counterproposal, or whether it is accepting or rejecting a proposal. A well-known example of such a protocol is the Contract Net (Smith, 1980). According to this protocol, agents decide upon their actions by exchanging demand and offer for services among themselves, together with status information.

\section{SPECIFICATION LANGUAGE $\chi$}

At Eindhoven University of Technology, department of Mechanical Engineering, a real-time concurrent programming formalism has been developed, called $\chi$. This formalism can be used for the specification and simulation of discrete industrial systems. It supports modularity and allows separate descriptions of the structure and of the components' behaviour. A specific feature of $\chi$ is the clear representation and unambiguous specification of interfaces between components (Mortel Fronczak, et al., 1995).

A system is treated as a collection of concurrently operating sequential components. A system component is modelled by a process as a sequential program where changes in the state of a process are accomplished by performing actions. Interaction between components is modelled by send and receive actions along fixed communication channels. A process is specified by a program in a CSP-like specification language preceded by Pascal-like declarations of local variables and statistical distributions. Processes do not share variables - they interact exclusively by using the communication and synchronisation primitives (synchronous message-passing). The reader may find an extensive example of the specification language $\chi$ in (Mortel Fronczak, et al., 1995; Chi, 1996). 


\section{MODEL FACTORY}

The model factory is a miniaturised, though still complex, model of a real Printed Circuit Board (PCB) assembly and test plant. The function of the model factory is to assemble and test pseudo PCBs. Each PCB consists of a board and a maximum of six components. Currently, two different types of boards and three types of components are used.

The model factory emulates operations which are performed on real PCBs during the manufacturing process. The operations of the model factory have been derived from case studies of real PCB manufacturing facilities. These operations are:

- screen printing: the bare PCB is positioned in the workstation, a PCB-specific screen is selected and moved into position, and a squeegee is reciprocated horizontally over the screen.

- component placement: the $\mathrm{PCB}$ is positioned in the workstation, and components are placed on the positions according to the componentplacement recipes for that product.

- reflow and cleaning: PCBs are passed through an oven and cleaning station

- test and repair: the PCB is inspected to see if it contains the components in the designated position, and component and functional tests are performed. If the PCB fails, it must be routed to an off-line diagnosis and repair workstation. Upon successful repair, the PCB is routed back to the test station.

In addition to the operations described above, the model factory contains some other features. Raw material and components are automatically supplied from a centralised raw material store and component store respectively. The model factory can support mixed model flow production, where different types of products can be manufactured at the same time. The model factory is designed for batch production, but the batch size can vary from batch to batch, as well as product to product. The maximum batch size in the model factory is three.

The process layout is depicted in Figure 1. The operations are indicated by square boxes, whereas stock points are indicated by triangles. The first stock point contains the two different empty board types. All products pass the screen printer, but alternative routings are possible between the two component placement stations. After the reflow and cleaning station, the batches may be stored in the in-

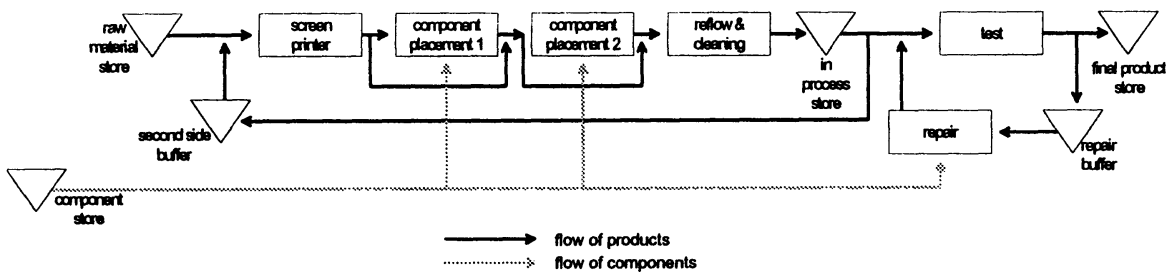

Figure 1 Primary process of the model factory 
process-store which consists of three locations for three products each. Here, a batch can be split or concatenated with other batches. Then, products are tested and - if necessary - repaired. In the test and repair cycle, a maximum of one batch can reside in the buffer. Finally, nine individual products can be stored in the finalproduct-store.

An additional feature is a loop from the in-process-store to the screen printer. This loop is necessary to manufacture PCBs that have components on both sides. These products have to pass the process twice, since only one side can be finished in one pass. The buffer in this second-side loop may contain only one batch (Timmermans, 1993a; Timmermans, 1993b).

\section{SPECIFICATION OF AN AGENT BASED CONTROL SYSTEM}

\subsection{Introduction}

During system design, decisions have to be made about the main properties of the control architecture. Using Cantamessa's work (1995) on the main features that characterise agent-based approaches, the following statements are made about the control architecture. The system being modelled is the model factory with a focus on the left part, i.e. between the screen printer and the reflow and cleaning station. The objects in the system are batches, workstations, and transportation devices. The objects in the system that act as agents are the workstations. The batches are passive entities flowing through the system, and the transportation devices act upon the orders of the workstations.

Furthermore, the agents' behaviour is derived from the Contract Net scheme with the possibility to subcontract jobs (see section 5.3). The system structure is heterarchical, i.e. all agents work on a level of complete parity, without any superior, coordinating entity. The information flow is directed via a network (see section 5.5). Agents do not have any knowledge of other agents' capabilities. Agents requesting a particular service pass their request via the network to all other agents connected to the network.

\subsection{Push Strategy}

Batches can be pushed through or pulled out of the factory. With a pull-approach, a planning is made in advance. The last station in the line, i.c. the final product store, is requested to deliver a batch of finished products at a certain due date. Then, the last station requests the appropriate batch from its preceding station, which - at its turn - asks for semi-finished batches to its predecessors, and so on. When a complete planning is made, the order is released and production starts. For an example of such a strategy, see (Wiendahl and Ahrens, 1995).

We have chosen for an opportunistic push-approach. With a push-strategy, the job is brought into the system at the first point of the line, namely the raw material 
store. Subsequently, the job finds its way through the system. However, in the model factory a convergent material flow is present at the component placement stations; both the boards and the components lead to these assembly stations. In general, since operation is not planned ahead, stock points should be created in order to decouple the main stream from the branches. These buffers can be replenished by means of a pull approach and simple inventory control heuristics. Just in front of the model factory's component placement stations, small buffers are located in which two component trays, each containing four components, are stored. If the first tray in a buffer is out of components, a new tray is ordered from the central component store. Upon arrival of the new tray with components at the buffer, the empty tray is removed from it.

The main advantage of an opportunistic dispatch method is that decisions concerning the distribution of work around the shop are based on the prevailing system status rather than on some projection of that status (as would be the case with a pull approach). Disadvantages include the fact that opportunistic schedulers are myopic, may ignore interactions with other components, and may only handle priorities in a rather cumbersome way (Upton, et al., 1991). Advantages and disadvantages of both approaches are displayed in Table 1.

Table 1 Advantages and disadvantages of the push and pull-approaches

\begin{tabular}{|c|c|c|}
\hline & Advantages & Disadvantages \\
\hline Push & $\begin{array}{l}\text { - Robust, capable of dealing } \\
\text { with disturbances } \\
\text { - Opportunistic behaviour, } \\
\text { routing flexibility }\end{array}$ & $\begin{array}{l}\text { - Less suitable for convergent } \\
\text { material flows } \\
\text { - Only short-term vision, } \\
\text { possibly myopic }\end{array}$ \\
\hline Pull & $\begin{array}{l}\text { Suitable for convergent } \\
\text { material flows } \\
\text { - Plans in the future }\end{array}$ & $\begin{array}{l}\text { - Excessive planning needed } \\
\text { - More sensitive to disturbances }\end{array}$ \\
\hline
\end{tabular}

\subsection{Protocol}

The basic assumption behind this agent-based system is that an agent does not have any knowledge about other agents. A workstation agent only has knowledge about the operation(s) the workstation is able to perform. However, this is paid for by extensive message traffic; for instance, when an agent is ready with a certain job, it has to send a task announcement to all agents.

The protocol is based on the Contract Net scheme. In the usual Contract Net protocol, batches push their way forward looking for resources (see e.g. (Lin and Solberg, 1992)). In our application, these decisions are entrusted to the agent controlling the resource from which the batch is to be set free. Workstation agents announce tasks, submit bids, and offer tasks. 
Furthermore, agents have the possibility to subcontract jobs. At their turn, subcontractors are not allowed to subcontract. An agent might divide a certain job among itself and other agents that are capable of fulfilling the same task. If, for instance, five components have to be placed, the component placement stations might divide the job in such way that component placer \#1 and component placer \#2 place two and three components respectively.

\subsection{Agent Structure}

An agent consists of six components: a request handler, a subcontracting component, a controller, a machine controller, a database, and a sender. The structure of an agent is shown in Figure 2. Note that arrows indicate communication between components rather than explicit message exchanges.

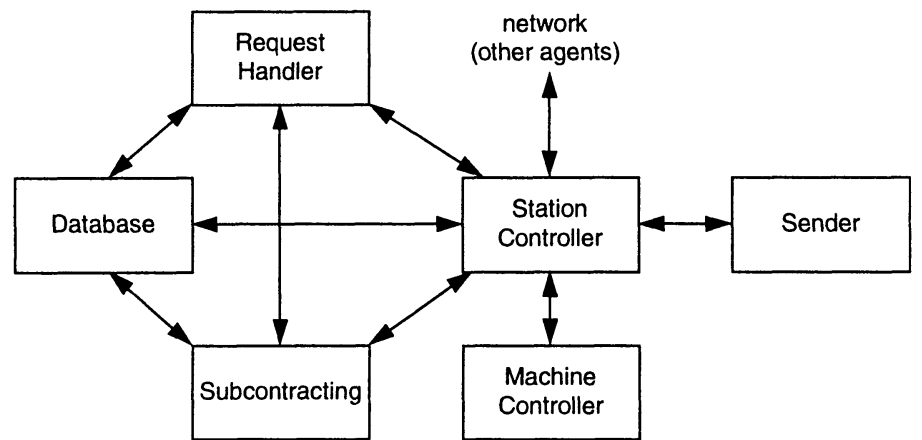

Figure 2 Logical diagram of agent components

The request handler issues bids as replies to incoming task announcements, whether they come from succeeding stations or from identical stations. In the latter case, the agent serves as a possible subcontractor. The request handler's task is to issue a bid. For this, it needs information from the database and possible subcontractors.

The subcontracting component puts subcontracts out to tender to other agents, in order to divide the current process step among the agent itself and other agents. If the agent is already a subcontractor, it will not try to put out to tender again; jobs are subcontracted once. Incoming bids are evaluated and the best bidder is rewarded with a task offer - if the overall bid is accepted. The subcontracting component sends the results of the bidding process to the request handler.

The station controller coordinates the agent's components. It also provides the interface with the outside world; all messages from/to other agents, such as negotiations between a predecessor and the request handler, pass through the controller. Furthermore, it commands the machine controller to start the operation on a batch. The machine controller performs the actual operation on the batch, which is evidently dependent on the type of station. 
The database stores run-time information of the agent. It sends information to the request handler, subcontracting component, and controller upon request. For example, in order to issue a bid, the request handler needs information about current work in progress, and about the components in the buffer - if appropriate.

The sender is responsible for the continuation of the batch. Before an operation on the batch is started, the sender sends task announcements to all agents, whether they are capable of performing the next process step or not. The sender receives incoming bids, evaluates them, and sends a task offer to the agent with the best bid. When the next agent has been selected, the sender notifies the controller about the destination of the batch.

\subsection{Communication Network}

A network is constructed for communication among agents. The network takes care for either a broadcast throughout the system in the case of a (sub-)task announcement, or for direct communication between two stations in all other cases. Rather than defining channels between every pair of agents, a network is designed that consists of network interfaces and a switch element (see Figure 3). A network interface is responsible for sending and receiving messages to/from the connected agent. The switch element connects the network interfaces. Upon reception of a message, it chooses an interface in order to transfer the message to the correct agent (Coenen, 1995).

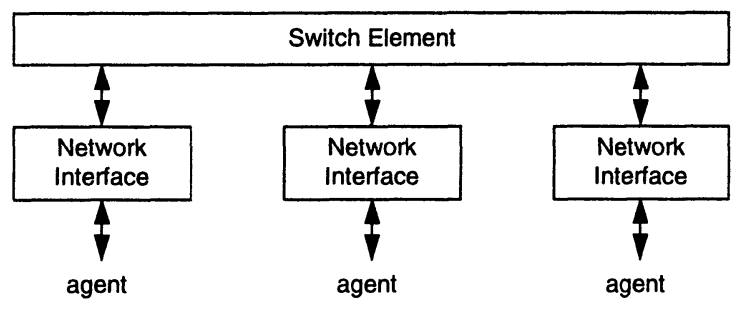

Figure 3 Communication network

\section{DISCUSSION}

In this section, we evaluate the agent based control system, and compare its characteristics with those of previously specified control architectures. A hierarchical and a heterarchical control system have been specified for the model factory (Timmermans, 1993b). We restrict ourselves to a comparison of the agent based control system with the heterarchical system only. In the latter system, routings are fixed and depend on the product type. Stations do not negotiate, but communicate with each other when they want to transfer a batch to the next station. 
Figure 4 shows some simulation results of both models. The horizontal axis shows the number of jobs simultaneously allowed in the factory, i.e. a type of input/output control. On the vertical axis, the average total completion time for ten samples of one hundred jobs is displayed. The throughput times of the agent based system are only slightly better than the throughput times of the heterarchical system. Whereas the heterarchical system does not have routing flexibility at all, the possibilities of the agent based system to avoid a busy station and direct the batch to a less busy station are limited. This is caused by the absence of alternative workstations, except for placing components.

The robustness of the agent based system is increased compared to the heterarchical system, due to the fact that routings were fixed in the latter system, whereas they are opportunistically 'composed' during operation in the agent based system. Again, the effect is only marginally, since in the present situation only the component placers can be interchanged to take care of malfunctions. If another station breaks down, the complete system will be blocked.

The flexibility, i.e. the modifiability of the agents, and the extensibility of the system, is better in the agent based system than in the heterarchical system. Stations in the heterarchical control system have knowledge about other stations. For instance, each station knows its direct 'neighbours'. When the factory is extended with a new workstation, the information its neighbours have of other stations needs to be updated. This is not necessary in the agent based system, since the agents communicate messages via the network. When a new agent is added to the system, the network is extended with a network interface that is connected to the new agent. Evidently, the switch component in the communication network of Figure 3 needs to be updated.

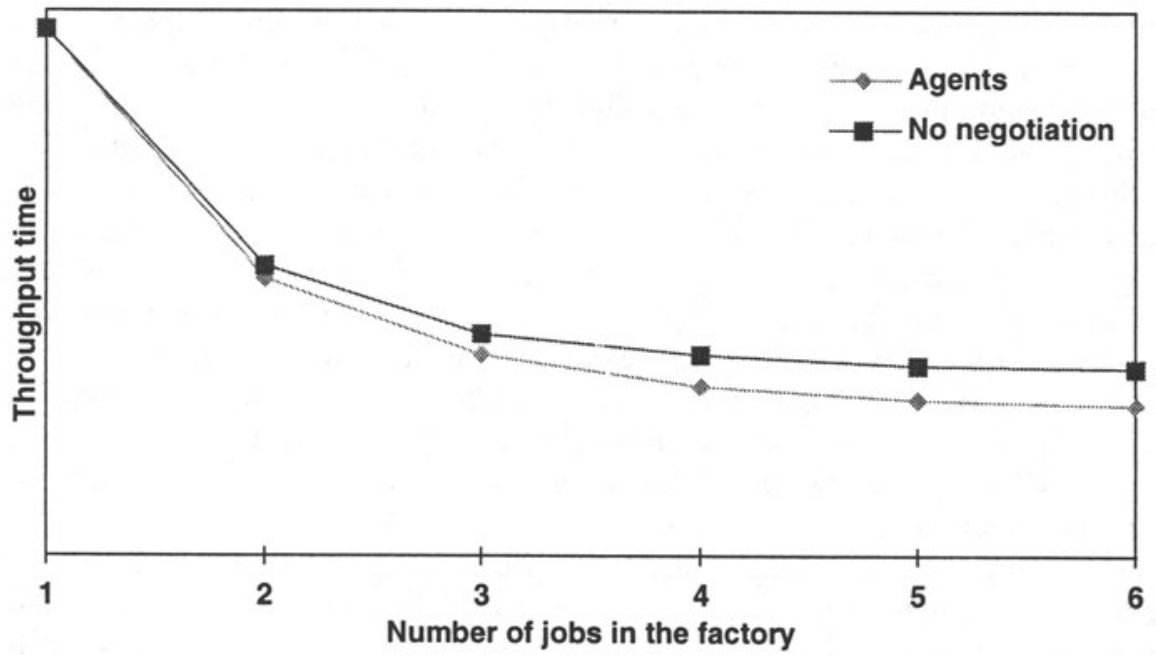

Figure 4 Simulation results 
Alternative approaches for a broadcast throughout the system are available. The drawback of a broadcast to all stations is that an overload of message exchanges may paralyse the system. An alternative solution would be to apply audience restriction, for instance by giving the agents local knowledge of other agents' skills. For an example, the reader is referred to (Cantamessa, 1995).

Another possibility to realise audience restriction is to give intelligence to the network. In the model factory, workstations communicate with each other via a network. An intelligent network might transport messages to appropriate agents only rather than to all agents. This network construction could easily be extended into a broker. Then, agents report finished jobs (i.e. idle workstations) and jobs to be executed to the broker, so the broker can match demand and supply of tasks.

The characteristics of the physical production system cause the agent based system to perform only slightly 'better' than the heterarchical system. This leads us to the conclusion that the model factory is not a suitable production system for the application of an agent based control system, and brings up the question in what situations agent based systems truly make a difference.

We introduce the concept of the routing space here to explain when agent based systems are most valuable and under which circumstances they can only have limited impact. The routing space is the set of possible transitions from one production station to another, and is product specific. Possible transitions can be specified as ordered pairs of station of origin and destination station. The more the routing space resembles a function, the less the agent based system is applicable. When the routing space is a function, at each station of origin a batch can only go to one station of destination. In this case, a deterministic schedule would suffice to exploit the 'full width' of the routing space, because there is no flexibility. That is, when a problem occurs, another station cannot be chosen. However, the more the number of ordered pairs in the routing space exceeds the number of process steps, i.e. from a station of origin a product can go to many stations of destination, the more the possibility to compose a schedule through negotiation at run time is going to be of value. In other words, agent based systems are more suitable in situations with many interchangeable workstations. However, in case of little uncertainty, a central scheduler would give more optimal routings and a better performance.

In case the process plan is not fixed, the size of the routing space is also determined by another factor. The less a certain order between operations is required, the more transitions from one station to another are possible. The routing space will increase correspondingly. Control in an 'orderless' situation, in terms of process plans, requires extensive memory capabilities to keep track of batch history. Given the characteristics of agents, they are less suitable for such an orderless situation.

For the same reason of lack of memory capabilities, agent based systems cannot cope with situations in which it might be more favourable to group operations and have them performed at one machine. Agents have a quite myopic view, which might lead to suboptimal routings. 
Last but not least, agents prove to be superior in a highly dynamic manufacturing environment with frequent changes in the production system. Adding or removing production stations only requires small modifications as compared to conventional control systems.

\section{CONCLUSIONS}

In certain situations, agent based production control systems provide a preferred alternative to the common architectural paradigms, such as the hierarchical production control architecture. The suitability of an agent based approach is dependent on characteristics of the production system, such as uncertainty in product specifications and process disturbances.

Future work in production control architecting will focus on the characteristics of various control architectures and the situations in which they are most appropriate. Also recently developed ideas, such as holonic manufacturing, the fractal factory, and bionic manufacturing, have to be taken into account.

\section{REFERENCES}

Cantamessa, M. (1995). A few notes upon Agent-based Modelling of Manufacturing Systems, in Proceedings of the CIM at Work conference (ed. J.C. Wortmann), pp. 301-317.

Chi. (1996). Example available at the Chi homepage. URL: http://www.tue.nl/wtb/wpa/se/chi/exam.htm

Coenen, F.W.J. (1995). A heterarchical control structure for flexible manufacturing systems (in Dutch). MSc Thesis Eindhoven University of Technology.

Jennings, N. R., P. Faratin, M.J. Johnson, P. O' Brien, and M.E. Wiegand. (1996). Using intelligent agents to manage business processes, in Proceedings of the First International Conference on The Practical Application of Intelligent Agents and Multi-Agent Technology (PAAM96), pp. 345-360. London, UK.

Lin, G.Y.-J., and J.J. Solberg. (1992). Integrated Shop Floor Control Using Autonomous Agents. IIE Transactions, 24(3), pp. 57-71.

Mortel-Fronczak, J.M. van de, J.E. Rooda, and N.J.M. van den Nieuwelaar. (1995). Specification of a Flexible Manufacturing System Using Concurrent Programming. Concurrent Engineering: Research and Applications, 3(3), pp. 187-194.

Smith, R.G. (1980). The Contract Net Protocol: High-Level Communication and Control in a Distributed Problem Solver. IEEE Transactions on Computers, 29(12), pp. 1104-1113.

Timmermans, P.J.M. (1993a). Modular Design of Information Systems for Shop Floor Control. PhD Thesis Eindhoven University of Technology. 
Timmermans, P. (1993b). Control architectures and modular information systems: a comparative experiment, in Proceedings of the international conference on Advances in Production Management Systems (APMS '93), (eds. I.A. Pappas and I.P. Tatsiopoulos), pp. 387-394. Elsevier Science Publishers.

Upton, D.M., M.M. Barash, and A.M. Matheson. (1991). Architectures and auctions in manufacturing. International Journal of Computer Integrated Manufacturing, 4(1), pp. 23-33.

Wiendahl, H.-P., and V. Ahrens. (1995). Knowledge-Based Support for Planning and Control in Distributed Production Systems, in Proceedings of the IFIP 5.7 Working Conference on Managing Concurrent Manufacturing to Improve Industrial Performance, pp. 429-443.

\section{BIOGRAPHY}

Arian Zwegers received his M.Sc. degree cum laude from the Eindhoven University of Technology in 1993. Currently, he is preparing his Ph.D. thesis at the same university. During two years, he was a member of the ESPRIT project VOICE. His research interests include shop floor control architectures, enterprise reference architectures and modular software design.

Henk-Jan Pels is an assistant professor in the Information Systems Group of the Department of Industrial Engineering at Eindhoven University of Technology. Since 1982, he has been researching and teaching databases and data modelling, especially for manufacturing applications. In 1988, he received his Ph.D. on a thesis on modular decomposition of data models. After that, he specialised in architectures for Computer Integrated Manufacturing and Engineering Data Management. He is also part time consultant for M.I.S. Organisatie Adviseurs.

Raymond Schrijver studied Technology and Society at the Eindhoven University of Technology (EUT) with a specialisation in Design Methodology. In 1993, he conducted a survey on Concurrent Engineering in the American industry for the Dutch Department of Economic Affairs. In 1995, he graduated in benchmarking for Concurrent Engineering. Currently, he is engaged in a two year advanced engineering program Computer Aided Design and Manufacturing at the Stan Ackermans Institute for Technological Design of the EUT.

Roelof van den Berg is a research program director with Baan Institute. He received his M.Sc. in Industrial Engineering and Management Science cum laude from Eindhoven University of Technology and will defend his dissertation at the same institution at the end of 1997. To date he has published over twenty articles on business modelling, business reengineering, quality management and the evolution of ERP-systems. Roelof is a member of IFIP's WG 8.2, ACM SIGMIS, IEEE Computer Society and the AIS. 